\title{
Visualization and Analysis of Data from Small-Body Missions with NASA's Solar System Treks Portals
}

Brian Day (1), Emily Law (2), Solar System Treks Development Team (2)

(1) NASA Solar System Exploration Research Virtual Institute. NASA Ames Research Center. Moffett Field, CA, USA.

(Brian.H.Day@nasa.gov)

(2) Jet Propulsion Laboratory, California Institute of Technology. Pasadena, CA, USA 91109. (Emily.S.Law@jpl.nasa.gov)

\begin{abstract}
The portals of NASA's Solar System Trek Project (SSTP) provide web-based suites of interactive visualization and analysis tools enabling mission planners, planetary scientists, students, and the general public to explore planetary surfaces as seen through the eyes of many different instruments aboard a wide range of spacecraft. The portals present a vast collection of mapped data products from past and current missions for a growing number of planetary bodies. As web-based toolsets, the portals do not require users to purchase or install any software beyond current web browsers. They feature a common user interface and are available through a single integrated website at https://trek.nasa.gov. This presentation will highlight 4 portals focusing on small bodies. We will provide an overview of established portals for (1) Ceres and (4) Vesta, and present the early capabilities for new portals being introduced for (162173) Ryugu and (101955) Bennu.
\end{abstract}

\section{Solar System Treks Overview}

NASA's Solar System Treks program of lunar and planetary mapping and modeling produces a suite of interactive visualization and analysis tools. The program is managed by NASA's Solar System Exploration Research Virtual Institute and developed at NASA's Jet Propulsion Laboratory. These tools enable mission planners, planetary scientists, and engineers to access mapped data products from a wide range of instruments aboard a variety of past and current missions, for a growing number of planetary bodies. While originally initiated for mission planning and science, this technology has demonstrated great benefits for public outreach.

The portals provide easy-to-use tools for browsing, data layering and feature search, including detailed information on the source of each assembled data product. Interactive maps, include the ability to overlay a growing range of data sets including topography, mineralogy, abundance of elements, and geology. They provide analysis tools that facilitate measurement and study of terrain including distance, height, and depth of surface features. They allow users to easily find and access the geospatial products that are available. Users have the ability to drill down to find the PDS data used to produce the geospatial products. Data products can be viewed in 2D and 3D, and can be stacked and blended together rendering optimal visualization that reveals details that no single data set can show. Data sets can be plotted and compared against each other. In addition to keyboard and mouse control, standard gaming and 3D mouse controllers allow users to maneuver first-person visualizations of flying across planetary surfaces. The portals also provide users the ability to specify any area of terrain for generation of STL/OBJ files that can be sent to 3D printers to make 3D models.

The new Virtual Reality Extension is an exciting addition to the Solar System Treks. Users can draw a path across the surface using the browser interface. A QR code is then generated which is read by the user's smart phone. Placing the phone in an inexpensive set of Google Cardboard-compatible goggles, the user then flies along their specified path in virtual reality.

Along with the web portals, the project supports additional clients, web services, and APIs that facilitate dissemination of planetary data to a range of external applications and venues.

As of abstract submission in May, 2019, six portals were available to the public to explore the Moon, Mars, Vesta, Ceres, Titan and IcyMoons (Saturn's moons Dione, Enceladus, Iapetus, Mimas, Phoebe, Rhea, and Tethys). Two new portals for Ryugu and Bennu are being prepared for initial public release. Additional portals for other bodies are in development and planning stages. 


\section{Vesta Trek and Ceres Trek}

In its investigations of Vesta and Ceres, NASA's Dawn mission has returned spectacular data detailing the surfaces of these two prominent small bodies in our Solar System's asteroid belt. In order to greatly facilitate dissemination, visualization, and analysis of this data, and public understanding of the mission, the Dawn mission has partnered with NASA's Solar System Treks Project (SSTP). SSTP has recently released an update to the Vesta Trek online portal (https://trek.nasa.gov/vesta/) and has released a new Ceres Trek portal (https://trek.nasa.gov/ceres/).

This presentation will showcase the use of the Ceres Trek and Vesta Trek portals and demonstrate how they can be used to visualize and analyze particularly interesting landforms such as the pitted terrain on Vesta and relic cryovolcanoes on Ceres. We will also demonstrate the new VR capability that has been added to the portals, allowing users to generate their own virtual reality flyovers for any user-defined paths along the bodies' surfaces.

\section{Ryugu Trek}

At the JpGU conference in May 2018, the Director General of the Japanese space program and the Chief Scientist of NASA announced that the two agencies would collaborate in the production of a new online portal hosting data from the Hayabusa2 mission. JAXA is providing mission data to SSTP, which is incorporating it into a new Ryugu Trek portal, currently in development. Both English and Japanese language interfaces are being produced. As the mission progresses, mission data will continue to be ingested into Ryugu Trek, facilitating visualization, dissemination, and analysis of data from Hyabusa2's historic encounter with this fascinating Near Earth Object.

\section{Bennu Trek}

While Hayabusa2 is exploring Ryugu, NASA's OSIRIS-REx mission is conducting a detailed examination of the asteroid Bennu, another Near Earth Object. At the request of NASA's Planetary Science Division and the OSIRIS-REx mission, SSTP began implementation a new portal for the asteroid Bennu, featuring data that is being returned from OSIRIS-Rex. The portal is being designed to facilitate outreach, mission planning, and planetary science. Together, Bennu Trek and Ryugu Trek provide an exciting capability to conduct comparative planetology with data being received from concurrent live missions.

\section{Looking Ahead}

Other small bodies are being considered as subjects for future Trek portals. As a technology demonstration, the SSTP team has developed a beta version of a portal for Comet 67P/ChuryumovGerasimenko using data from the European Space Agency's Rosetta mission. Other Near Earth Asteroids are also candidates for future portals including (433) Eros utilizing data gathered by the NEAR Shoemaker mission, and (25143) Itokawa using data from JAXA's Hayabusa mission. Together, a growing collection of small body Trek portals would enhance capabilities for comparative planetology among this fascinating class of objects. They could also be useful, from both science and outreach perspectives, for Planetary Defense efforts being planned by NASA and its international partners.

\section{Summary and Conclusions}

NASA's Solar System Treks Project provides online, web-based visualization portals that facilitate data dissemination, visualization, and analysis for a variety of planetary bodies. With the portals implemented for Vesta, Ceres, Ryugu, and Bennu, these capabilities have been extended to our Solar System's small bodies. The EPSC community is invited to provide suggestions and requests as the development team continues to expand the capabilities of the portals.

\section{Acknowledgements}

The authors would like to thank the Planetary Science Division of NASA's Science Mission Directorate, NASA's SMD Science Engagement and Partnerships, the Advanced Explorations Systems Program of NASA's Human Exploration Operations Directorate, and the Moon to Mars Directorate for their support and guidance in the development of the Solar System Treks. 